THE KURUME MEDICAL JOURNAL

Vol. 38, p. 117-122, 1991

\title{
Zero Operative Mortality for Gastric Cancer with Hepatic Cirrhosis
}

\author{
JINRYO TAKEDA, KEN HASHIMOTO, TAKAHO TANAKA, KIKUO KOUFUJI, \\ HIDEAKI YAMANA AND TERUO KAKEGAWA
}

Department of Surgery, Kurume University School of Medicine, Kurume, 830 Japan

Received for publication April 2, 1991

\begin{abstract}
Summary: Thirty-two cases of gastric cancer associated with hepatic cirrhosis were reviewed, retrospectively. These patients represented $1.3 \%$ of 2590 patients with gastric cancer who were treated over the 23 year period from 1966 to 1988 . The male to female ratio was $9.7: 1$ for gastric cancer patients with hepatic cirrhosis (mean age, 63.2 years) and $2: 1$ for all patients with gastric cancer (mean age, 61.2 years). Among the 32 patients, one was inoperable, and 31 patients underwent surgical exploration. The gastric cancers were resected in 28 patients $(90.3 \%)$, while the cancers in 3 patients (9.7\%) were non-resectable. Twenty-two patients underwent curative resections with a five-year survival rate of $51 \%$. Postoperative complications occurred in 6 patients (20\%), but no anastmotic leakage occurred. There were no operative mortalities. On the basis of the present study, different surgical procedures are indicated depending on the preoperative hepatic conditions for patients with gastric cancer accompanied by hepatic cirrhosis.
\end{abstract}

Keywords: Gastric cancer - hepatic cirrhosis - gastrectomy - operative indication - complication - mortality

\section{Introduction}

Among the various treatments, gastrectomy provides the best long-term survival in patients with gastric cancer. However, gastrointestinal surgery for patients with hepatic cirrhosis is associated with a high operative morbidity and mortality. Therefore, an accurate preoperative assessment of the risk from the operation is important in gastric cancer patients with hepatic cirrhosis.

The purpose of this study is to report on the gastric cancers in 32 patients with hepatic cirrhosis and to propose selection criteria for different surgical interventions.

\section{Patients and Methods}

During the 23 years from 1966 to 1988 , a total of 2590 gastric cancer patients were referred to Kurume University Hospital for surgery. Thirty-two of these patients $(1.3 \%)$ had hepatic cirrhosis, which was histologically confirmed by preoperative and/or intraoperative liver biopsies. The male to female proportion of the 32 patients with hepatic cirrhosis was significantly higher $(p<0.001)$ than the male proportion of all 2590 gastric cancer patients. The male to female ratio was 9.1:1 for patients with hepatic cirrhosis, as compared to $2: 1$ for all gastric cancer patients. The mean age of the patients with hepatic cirrhosis was 63.2 years, while that of all patients was 61.2 years (not statistically different).

The laboratory and clinical tests that were used for the preoperative assessment of operative risk for patients with hepatic cirrhosis are listed in Table 1 . These included K-ICG (clearance rate of indo- 
TABLE 1

Preoperative assessment of operative risk

\begin{tabular}{l|c|c|c}
\hline \multirow{1}{*}{ Grade } & Low risk & Medium risk & High risk \\
\cline { 2 - 4 } & I & II & III \\
\hline KICG (min-1) & $>0.080$ & $>0.060$ & $<0.060$ \\
ICG Rmax (mg/kg/min) & $>0.60$ & $>0.45$ & $<0.45$ \\
HPT (\%) & $>70$ & $>55$ & $<55$ \\
PTT (\%) & $>75$ & $>65$ & $<65$ \\
Ch-E (International unit) & $>400$ & $>350$ & $>250$ \\
50g OGTT & Normal Parabolic & Parabolic & Parabolic $\sim$ Linear \\
Albumin (g/dl) & $>3.2$ & $>3.0$ & $<3.0$ \\
Ascites & $(-)$ & $(-)$ & $( \pm)$ \\
\hline
\end{tabular}

TABLE 2

Locations of the gastric cancers

\begin{tabular}{c|c|c}
\hline Main Location & No. of Cases & Percent \\
\hline C & 7 & 21.9 \\
M & 14 & 43.8 \\
A & 11 & 34.3 \\
\hline
\end{tabular}

cianine green), ICG-Rmax (maximal removal rate of ICG), HPT (hepaplastin test), PTT (prothrombin time), OGTT (oral glucose tolerance test) and the presence or absence of ascites. Based on the results of these tests, the patients were classified into one of three groups; low, medium and high risk (Table 1).

The locations of the gastric cancers and the number of patients for each location are summarized in Table 2.

\section{Results}

One of 32 gastric cancer patients with hepatic cirrhosis was judged to be inoperable due to the advanced stages of the cancer and cirrhosis. Surgical exploration was performed in the remaining 31 patients. Twenty-eight patients $(90.3 \%)$ underwent gastric resection, while 3 pa-
TABLE 3

Results of preoperative liver function tests

\begin{tabular}{l|c|c}
\hline \multirow{2}{*}{ Liver Function } & \multicolumn{2}{|c}{ Group } \\
\cline { 2 - 3 } & $\begin{array}{c}\text { Resection } \\
(\mathrm{n}=28)\end{array}$ & $\begin{array}{c}\text { Non-Resection } \\
(\mathrm{n}=4)\end{array}$ \\
\hline Total Bilirubin & $1.12 \pm 0.678$ & $0.78 \pm 0.26$ \\
ICG (15 min.) & $18.79 \pm 11.18$ & $11.80 \pm 9.41$ \\
TTT & $10.97 \pm 10.40$ & $12.21 \pm 9.85$ \\
ZnTT & $15.75 \pm 9.54$ & $18.31 \pm 7.54$ \\
GOT & $64.10 \pm 38.90$ & $59.60 \pm 48.30$ \\
GPT & $37.40 \pm 26.90$ & $40.20 \pm 37.60$ \\
Al-p & $11.88 \pm 5.67$ & $12.74 \pm 9.32$ \\
$\gamma$-GTP & $34.39 \pm 14.67$ & $32.44 \pm 22.36$ \\
Total Protein & $6.80 \pm 0.72$ & $7.20 \pm 0.89$ \\
Albumin & $3.48 \pm 0.49$ & $3.50 \pm 0.46$ \\
\hline
\end{tabular}

tients $(9.7 \%)$ were managed with palliative non-resectional procedures.

The results of preoperative liver function tests are summarized in Table 3. There was no statistical difference in liver function between the resection group $(n=28)$ and non-resection group $(n=4)$. Preoperative renal function tests i.e., 15 min. PSP (phenolsulfonphtalein) test, BUN (blood urea nitrogen) and creatinine are summarized in Table 4 . There was no statistical difference between the two groups.

Fifteen patients were class-A by Child's 
TABLE 4

Pre-operative renal function

\begin{tabular}{l|c|c}
\hline Test & $\begin{array}{c}\text { Resection } \\
(\mathrm{n}=28)\end{array}$ & $\begin{array}{c}\text { Non-Resection } \\
(\mathrm{n}=4)\end{array}$ \\
\hline PSP (15) & $30.18 \pm 13.40$ & $50.50 \pm 0.71$ \\
BUN & $18.07 \pm 8.29$ & $22.60 \pm 17.03$ \\
Creatinine & $1.01 \pm 0.45$ & $1.72 \pm 0.82$ \\
\hline
\end{tabular}

TABLE 5

Child's classification and operation type

\begin{tabular}{l|r|r|r}
\hline \multirow{2}{*}{ Operation } & \multicolumn{3}{|c}{ Child } \\
\cline { 2 - 4 } & A & B & C \\
\hline Total Gastrectomy & 2 & 1 & 0 \\
Lower Esophagectomy plus & 1 & 0 & 0 \\
Total Gastrectomy & 1 & 1 & 1 \\
Proximal Gastrectomy & 10 & 9 & 2 \\
Distal Gastrectomy & 1 & 0 & 1 \\
Bypass & 0 & 1 & 0 \\
Laparotomy & 0 & 0 & 1 \\
Inoperable & 15 & 12 & 5 \\
\hline \multicolumn{1}{c|}{ Total } & &
\end{tabular}

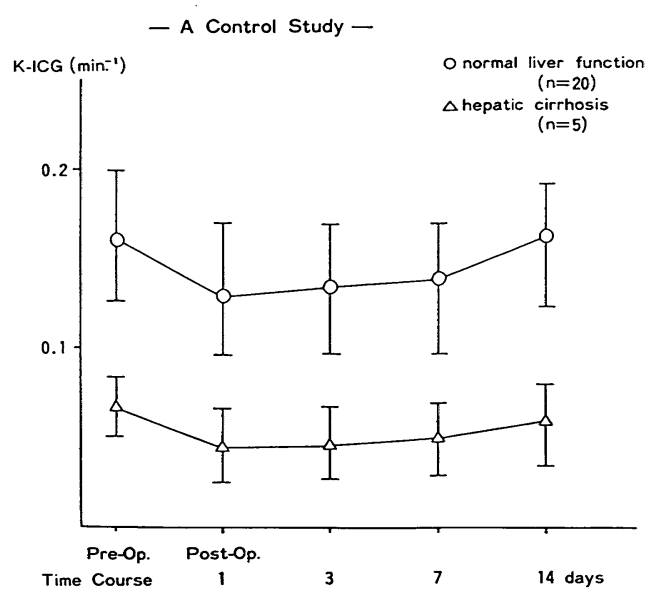

Fig. 1 Pre- and post-operative clearance rate of indocyanine green from blood $\{\mathrm{K}$ ICG $\left.\left(\min ^{-1}\right)\right]$ in patients with gastric cancer. classification, 12 patients were class-B, and 5 patients were class-C (Table 5 ).

The K-ICG was 0.078 , preoperatively, and this decreased markedly to 0.038 at 1 day postoperatively in 5 hepatic cirrhosis patients. However, in the normal liver function gastrectomized control group, the K-ICG was 0.16 preoperatively, and returned to the preoperative levels by 14 days postoperatively (Fig. 1).

The patients were also graded according to a preoperative assessment of operative risk, as shown in Table 1 . Sixteen patients were included in grade-I, 13 in grade-II; and 3 patients in grade-III. Two of 3 grade- III patients naturally developed a large porto-caval venous shunt, with $\mathrm{K}$-ICG values less than 0.45 and hyperammonemia.

The operative procedures performed for patients in the different grades are summarized in Table 6. Two grade-III patients underwent gastrectomies ( 1 proximal and 1 distal) with ligation and division of the large shunt. Splenectomy was performed in 3 of 4 patients who had total gastrectomies, in all 3 patients who had proximal gastrectomies, and in 3 of 21 patients who had distal gastrectomies preserving the short gastric vessels.

\section{TABLE 6}

Preoperative grade of hepatic cirrhosis and operation type

\begin{tabular}{c|r|r|r}
\hline \multirow{2}{*}{ Operation } & \multicolumn{3}{|c}{ Grade } \\
\cline { 2 - 4 } Total Gastrectomy & I & II & III \\
\hline Including lower esophagectomy) & 4 & 0 & 0 \\
Proximal Gastrectomy & 1 & 1 & 1 \\
Distal Gastrectomy & 10 & 10 & 1 \\
Bypass & 1 & 1 & 0 \\
Laparotomy only & 0 & 1 & 0 \\
Inoperable & 0 & 0 & 1 \\
\hline Total & 16 & 13 & 3 \\
\hline
\end{tabular}


The stage of gastric cancer in 32 patients and the resectability of the cancer in each stge are listed in Table 7.

Postoperative complications occurred in 6 of 31 surgical patients; the rate of complications were 20\% (Table 8). How ever, no anastomotic leakage was en-

TABLE 7

Cancer stage and resectability

\begin{tabular}{c|c|c}
\hline stage & No. of Cases & Resections (\%) \\
\hline I & 17 & 100 \\
II & 2 & 100 \\
III & 3 & 100 \\
IV & 10 & 60 \\
\hline
\end{tabular}

TABLE 8

Post-operative complications

\begin{tabular}{l|c}
\multicolumn{1}{c|}{ Variables } & No. of Cases \\
\hline Bleeding & 1 \\
Ascites (severe) & 2 \\
Hepatic Coma & 1 \\
Pneumonia & 1 \\
Renal Failure & 1 \\
Chronic Encephalopathy & 2 \\
Anastomotic Leakage & 0 \\
\hline
\end{tabular}

Occurrence of complications

$6 / 31 \times 100=19.4(\%)$

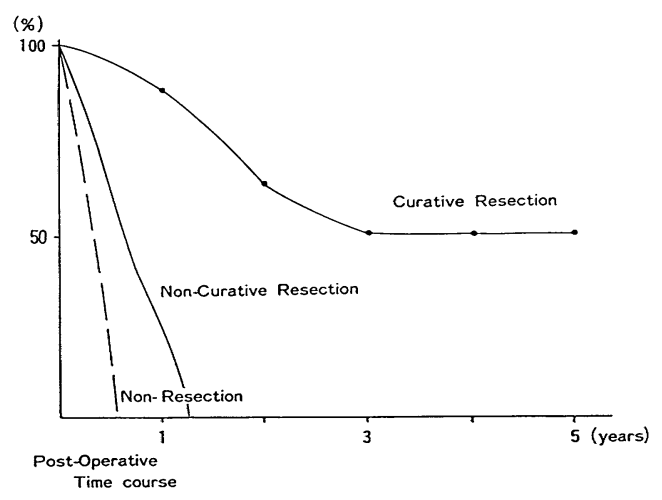

Fig. 2 Cumulative 5-year survival rates for each type of operation. countered. In addition, there were no postoperative mortalities in the present study. The five year survival rate with the Kaplan-Meier method in patients who underwent curative resecions $(n=22)$ was $51 \%$ (Fig. 2). These patients died mainly from cirrhosis-related causes, such as hepatic failure or hepatoma.

\section{Discussion}

The prognosis following surgery for gastric cancer has markedly improved as the result of earlier diagnosis, advances in surgical techniques, and pre- and post-operative management. However, gastrointestinal surgery in the presence of hepatic cirrhosis has a high operative morbidity and mortality. Mortality rates of $20-30 \%$ have been reported recently (Garrison et al. 1984; Fekete et al. 1987; Sirinek et al. 1987). Fisher (1983) has suggested that general anesthesia causes a $20 \%$ decrease in hepatic blood flow rate, which could affect the mortality.

Garrison et al. (1984) reported that infection and/or contamination after operation in hepatic cirrhosis patients were associated with a high mortality rate of $64 \%$ when infection was present, as compared to $21 \%$ when infection was absent. Operative urgency was directly related to the mortality. Dobernech et al. (1983) reported a mortality rate of $45 \%$ following emergency procedures in non-bleeding hepatic cirrhosis patients, while it was only $11 \%$ following elective operations.

Stone (1977) reported that Child's classification was initially designed to predict the operative risk for patients undergoing a porto-caval systemic shunt operation, the classification was also useful in predicting the outcome following other major surgical procedures. Garrison et al. (1984) also reported a correlation of the Child's classification to the operative mortality. Their mortality rate was $10 \%$ in 50 class- 
A patients, $31 \%$ in 29 class-B patients, and $67 \%$ in 21 class-C patients. Furthermore, elevated bilirubin, hypoalbuminemia, and presence of ascites were found to be significantly different between survivors and non-survivors in other studies of non-shunt ceriotomies in cirrhotic patients (Schwartz, 1981). Coagulation parameters could also be correlated with survival (Aranha, 1982). Because various factors affect the out come of gastric surgery in patients with hepatic cirrhosis, appropriate preoperative assessment is of paramount importance to determine the indication and the type of operative procedures. The selection of the operation should be based on both cancer stage and hepatic function. In the present evaluation of 32 patients, only one patient was inoperable and three patients had unresectable cancers, however, the remaining 28 patients were managed with gastrectomies. In terms of Child's classification, 14 of 15 patients of class-A and 11 of 12 patients of class-B underwent surgery, including total, proximal and distal gastrectomies. Two other patients in these classes had unresectable cancers of stage IV. Even 3 of 5 patients in class-C were treated by gastric resections, although one patient had an unresectable stage IV cancer and another patient was inoperable due to severe hepatic dysfunction in addition to stage IV cancer.

Gastric resection for cancer can usually be conducted without remarkable risk in patients with mild hepatic dysfunction such as patients without increased bilirubin, ascites or encephalopathy. Preoperative laboratory criteria that are generally acceptable for gastrectomy include total serum bilirubin of $2 \mathrm{mg} / \mathrm{dl}$ or less, GOT and GPT less than 150 units, total serum albumin more than $3.0 \mathrm{~g} / \mathrm{dl}$, KICG more than 0.06 , ICG-Rmax more than 0.45 , HPT more than 55\%, PTT more than $65 \%$ and ch-E more than 250 units.

In the present study, the preoperative assessment criteria of operative risk were established, as shown in Table 1 . The patients were classified on the basis of operative risk into three grades. This grading requires more detailed information on hepatic function than Child's classification. On the basis of the 32 patients with varying grades, selection criteria can be proposed for the surgical procedures. For patients of grade-I (low risk), a radical extended total or subtotal gastrectomy including lymph node dissection should be performed. For patients of grade-II (medium risk), a radical subtotal gastrectomy and lymph node dissection or a total gastrectomy without lymph node dissection should be performed. For patients of grade-III (high risk), a palliative bypass operation or non-operation management should be provided. Non-operative management includes endoscopic laser therapy for early stage cancer. When esophageal varices are present, esophageal transection using a stapler can be added to the gastrectomy procedure to prevent postoperative bleeding from the varices. In this situation, preoperative sclerotherapy for varices is contraindicated because of esophageal damage caused by the sclerosing agents (Takeda et al. 1989).

With these management and surgical procedures, excellent results were achieved in the present series. Although postoperative complications occurred in about $20 \%$ of the patients, this rate is not high for patients with combined gastric cancer and hepatic dysfunction. This study indicates that gastric cancer in association with hepatic cirrhosis can be successfully managed surgically with appropriate preoperative assessment of hepatic conditions, with proper selection of the type of surgical procedure, and with careful perioperative management. The presence of hepatic cirrhosis alone is no longer a contraindication for gastrectomy in patients with gastric cancer. 


\section{References}

Aranha, G. B., Sontag, S. J. and Greenlee, H.B. (1982). Cholecystectomy in cirrhotic patients: a formidable operation. Am. J. Surg. 143, 55-60.

Doberneck, R.C., Sterling, W.A. and Allison, D.C. (1983). Morbidity and mortality after operations in nonbleeding cirrhotic patients. Am. J. Surg. 146, 306-309.

Fekete, F., Belgihiti, J., Cherqui, D., Longonnet, F. and GAYET, B. (1987). Results of esophagogastrectomy for carcinoma in cirrhotic patients. Ann. Surg. 206, 74-78.

Fischer, J.E. (1983). Current concepts of surgery in portal hypertension. Semin. Liver Dis. 3, 306-309.

Garrison, R.N., Cryer, H.M., Howard, D. A. and Polk, H.C. (1984). Clarification on risk factors for abdominal operations in patients with hepatic cirrhosis. Ann. Surg. 199, 648-
655.

Japanese Research Society for Gastric Cancer: The general rules for gastric cancer study in surgery and pathology. (1973). Jpn. J. Surg. 3, 61-71.

SchwARTz, S.I. (1981). Biliary tract surgery and cirrhosis: a critical combination. Surgery 90, 577-583.

Sirinek, K. R., Burk, R.R., Brown, M. and Levine, B.A. (1987). Improving survival in patients with cirrhosis undergoing major abdominal operations. Arch. Surg. 122, 271-273.

Stone, H.H. (1977). Preoperative and postoperative care. Surg. Clin. North Am. 57, 409-419.

Takeda, J. Hashimoto, K., Tsuji, Y., Tanaka, T., Yasumoto, K., Yamana, H., Kakegawa, T. and AraKI, T. (1989). Gastric operation in patients with hepatic cirrhosis following endoscopic sclerotherapy. Kurume Med. J. 36, 81-85. 